\title{
Unconjugated bilirubin from cord blood; an indicator for prophylactic phototherapy
}

\author{
Kondekar $\mathbf{A}^{1}$, Kondekar $\mathbf{S}^{\mathbf{2}}$ \\ Dr Alpana Kondekar, Dr Santosh Kondekar, Both are Associate Professor, Department of Pediatrics, TN Medical \\ College Mumbai India
}

Address for correspondence: Dr Santosh Kondekar, Email: writetodoctor@ gmail.com

\begin{abstract}
Neonatal hyperbilirubinemia is a cause of concern for the parents as well as for the pediatricians as it is a leading cause behind readmission to hospital after the early discharge of mother-baby dyad.
\end{abstract}

Keywords: Unconjugated bilirubin, Cord bilirubin, Prophylactic phototherapy

Neonatal hyperbilirubinemia is very common condition during neonatal period presenting clinically as jaundice and is caused by certain metabolic and genetic defects resulting in transient bilirubin conjugation deficiency, including hepatic uptake and intracellular transport deficiency, and increased enterohepatic circulation [1].

Neonatal hyperbilirubinemia is a cause of concern for the parents as well as for the pediatricians as it is a leading cause behind readmission to hospital; after the early discharge of mother-baby dyad. Kernicterus as a sequelae of severe jaundice is a well known entity but a large group of children manifest with syndrome of bilirubin-induced neurologic dysfunction [BIND] that represents varied degree of neuro-motor manifestations extend to a range of subtle processing disorders with objective disturbances of visual-motor, auditory, speech, cognition, and language to encephalopathy [2].

An estimated $50 \%$ of term and $80 \%$ of preterm infants develop jaundice, typically 2-4 days after birth [3]. The early recognition of jaundice clinically by the parents and care givers is very difficult. Severe jaundice, and even kernicterus, can occur in an otherwise healthy term baby in presence of risk factors. Identification of the risk factors before discharging the newborn from hospital should be a routine practice. In recent years; many efforts have been made to identify infants at risk of neonatal jaundice that can reduce hospital stay for normal babies and identify significant hyperbilirubinemia that may happen in the future. Various strategies to predict significant hyperbilirubinemia are : 1 . close follow-up within 1-2 days of early discharge, 2. umbilical cord bilirubin concentration at birth, 3.routine pre-discharge serum bilirubin and transcutaneous bilirubin measurement, as well as 4.the universal clinical assessment of risk factors of developing jaundice [4]. Cord bilirubin estimation is a noninvasive and reliable technique for predicting hyperbilirubinemia. Several studies have shown a significant correlation between cord blood bilirubin level and subsequent hyperbilirubinemia. Critical cord blood bilirubin level $>2.5 \mathrm{mg} / \mathrm{dl}$ has a high probability to develop significant hyperbilirubinemia [5].

The number of jaundiced newborns undergoing phototherapy was significantly higher with cord bilirubin level $>2.3 \mathrm{mg} / \mathrm{dl}$ than those newborns with cord bilirubin levels $<2.3 \mathrm{mg} / \mathrm{dl}$ [5].

An Egyptian study concluded that the strongest predictor of receiving phototherapy was total cord bilirubin compared to gestational age, ABO, incompatibility, RH incompatibility and sex. Serum bilirubin in cord blood was indicative of jaundice severity during the first week of life. Levels that were equal to or greater than $2.05 \mathrm{mg} / \mathrm{dl}$ and 2.15 in PT and FT respectively indicate the need for further treatment by phototherapy. In addition, it was also concluded that the presence of mother/baby blood group incompatibility was statistically significant for the occurrence of high total cord bilirubin that was indicative for phototherapy treatment [6].

An Iranian study had similar results with cut off for critical bilirubin level was $2 \mathrm{mg} / \mathrm{dl}$ while Agarwal et al's study which showed that if the amount of total bilirubin in infant's serum is $<6 \mathrm{mg} / \mathrm{dl}$ within the first $24 \pm 6$ hours after birth, this infant will not have pathological 
jaundice, with a sensitivity $95 \%$, specificity of $70.6 \%$, supporting the cord blood can be a good predictor for subsequent hyperbilirubinemia $[7,8]$.

Hour specific percentile charts based on serum bilirubin at different postnatal ages are available and subsequent hyperbilirubinemia can be predicted with reasonable accuracy by plotting $\mathrm{hr}$ specific bilirubin on these charts. A TSB level $6 \mathrm{mg} / \mathrm{dl}$ within $21 \mathrm{hrs}$ of birth has a high predictive value for hyperbilirubinemia later [8]. A study from Brazil; noted that $53 \%$ of normal newborns with unconjugated cord bilirubin levels more than $2 \mathrm{mg} / \mathrm{dl}$ had indication for phototherapy on day 3 of life; also it was more likely in those with blood group incompatibilities [9] Cord blood bilirubin could be a useful indicator of developing jaundice in newborns and the use of cut off cord bilirubin levels could be a useful predictor of significant perbilirubinemia. The use of the cut-off cord unconjugated bilirubin levels of $2.0 \mathrm{mg} / \mathrm{dl}$ in all healthy late pre-term and full-term newborns could be a useful predictor of significant hyperbilirubinemia that will need phototherapy and avoid the risk of severe hyperbilirubinemia that may need exchange transfusion. Also it will be a major step in prevention of bilirubin-induced neurologic dysfunction in children in whom late recognition or late intervention of hyperbilirubinemia in neonatal period was a major cause for such deficit.

The current AAP guidelines recommends the use of the total bilirubin concentration/albumin (TBC/A) ratio in addition to the TBC. Umbilical cord serum albumin levels also; are useful in predicting subsequent neonatal jaundice in healthy term newborns [10]. Menon $\mathrm{M}$ et al in this issue found in her study that A cord bilirubin level above 2.05 can predict the need for treatment. [11]

\section{References}

1. Watchko JF, Lin Z. Genetics of neonatal jaundice. Stevenson DK, Maisels MJ, Watchko JF. Care of the jaundiced neonate. New York: McGraw-Hill; 2012. 127.

2. Johnson L, Bhutani VK. The clinical syndrome of bilirubin-induced neurologic dysfunction. InSeminars in perinatology 2011 Jun 30 (Vol. 35, No. 3, pp. 101-113). WB Saunders.
3. Woodgate P, Jardine LA. Neonatal jaundice: phototherapy. BMJ Clin Evid. 2015 May 22. 2015

4. Rostami N, Mehrabi Y. Identifying the newborns at risk for developing significant hyperbilirubinemia by measuring cord bilirubin levels. J Arab Neonatol Forum 2005;2:81-5.

5. Satrya R, Effendi SH, Gurnida DA. Correlation between cord blood bilirubin level and incidence of hyperbilirubinemia in term newborns. Paediatr Indones. 2009 Nov;49:349-54.

6. Zeitoun AA, Elhagrasy HF, Abdelsatar DM. Predictive value of umbilical cord blood bilirubin in neonatal hyperbilirubinemia. Egyptian Pediatric Association Gazette. 2013 Jan 31;61(1):23-30.

7. Ghamsari AA, Mohamadzadeh A, Khodadadi A, Rezaei M. Does umbilical cord bilirubin level have predictive value in pathologic neonatal hyperbilirubinemia?. Iranian Journal of Neonatology IJN. 2013 May 16;4(1):32-5.

8. Agarwal R, Kaushal M, Aggarwal R, Paul VK, Deorari AK. Early neonatal hyperbilirubinemia using first day serum bilirubin level. Indian pediatrics. 2002 Aug;39(8):724-30.

9. Bernaldo AJ, Segre CA. Bilirubin dosage in cord blood: could it predict neonatal hyperbilirubinemia?. Sao Paulo Medical Journal. 2004 May;122(3):99-103.

10. Meena K J, Singh S, Verma R C, Sharma R. Utility of Cord Blood Albumin as a Predictor of Significant Neonatal Jaundice in Healthy Term Newborns. Pediatric Oncall [serial online] 2015[cited 2015 October-December 1];12. Art \#66. Available From : http://www.pediatriconcall.com/Journal/Article/FullTex t.aspx artid=988\&type $=$ J\&tid $=\&$ imgid $=\&$ reportid $=523$ \&tbltype $=$

11. Maya Menon, Sreejyothi G, Raveendranath K, Incidence of early neonatal hyperbilirubinemia in ABO incompatibility and cord bilirubin as a predictor for phototherapy. Paed Rev: Int J Pediatr Res 2016;3(4):218-224.doi:10.17511/ijpr.2016.4.02.

\section{How to cite this article?}

Vanza B, Patel U, Kulkarni, Khare N' Bilateral TMJ ankylosis, anesthetic and surgical challenge- case report. Pediatr rev. Int J Pediatr Res 2016;3(4):212-213. doi:10.17511/ijpr.2016.i04.01 